\title{
Uso del midazolam en el control y manejo de la ansiedad en niños durante la atención odontológica: una revisión bibliográfica
}

\author{
Use of Midazolam in anxiety control and management of of children during den- \\ tal care: literature review
}

Patricio Tello Aros ${ }^{1}$, Sergio Herrera Bustamante' ${ }^{1}$ Katerin Terán Quezada², Daniela Rojas Cáceres³

Para citar este artículo: Tello PF, Herrera S, Terán K, Rojas D. Uso del Midazolam en el control y manejo de la ansiedad en niños durante la atención odontológica: Una revisión bibliográfica. Ustasalud; 2017;16: 69-77

Licencia Creative Commons

\section{(c) (1) $(\Theta$} lo tanto, los lectores pueden acceder libremente a los artículos en su formato .pdf, igualmente podrán descargarlos y difundirlos; sin embargo no podrán modificarlos o alterarlos, adicionalmente se debe reconocer la autoría de las personas que figuran en las publicaciones, pero estas no podrán comercializadas.

\section{RESUMEN}

Cuando las técnicas de manejo del comportamiento en niños no son suficientes puede ser necesario utilizar técnicas avanzadas como la sedación consciente; uno de los fármacos más usados para este propósito es el Midazolam. El objetivo de este estudio fue conocer la evidencia científica actual sobre la efectividad del Midazolam para su uso en odontopediatría.

Para llevar a cabo este estudio se consultaron bases de datos como Pubmed, Scielo, EBSCOhost, Cochrane Library y Google Académico, teniendo como límites de búsqueda documentos publicados entre el 2005 y el 2015 en idioma inglés o español. Se consideraron artículos de revisión, ensayos clínicos y estudios de series de caso.

Se concluyó que la sedación con Midazolam, es una técnica válida, segura y eficiente para controlar la ansiedad en niños siempre y cuando sean respetados los regímenes de administración propuestos y contar con el equipamiento y conocimiento necesarios.

Palabras clave: Ansiedad dental, sedación consciente, midazolam, odontopediatría.

\section{ABSTRACT}

In cases where behavior management techniques are not enough, it may be necessary to use more advanced techniques such as conscious sedation. One of the most commonly drugs used for this purpose is Midazolam. The aim of this study was to review the current scientific evidence on the effectiveness of Midazolam for its use in pediatric dentistry.

To carry out this study, databases such as Pubmed, Scielo, EBSCOhost, Cochrane Library and Google Scholar were used, only documents published between 2005 and 2015 in English or Spanish were considered. Review papers, clinical trials and case series studies were included.

It was concluded that sedation with Midazolam is a valid, safe and efficient technique to control anxiety in children if administration regimens are respected and the necessary equipment and proper knowledge.

Keywords: dental anxiety, conscious sedation, midazolam, pediatric dentistrty

\begin{abstract}
${ }^{1}$ Centro de Salud Familiar (CESFAM) de Alhué, Servicios de Atención Primaria en Salud, Alhué, Chile

${ }^{2}$ Modulo Dental Junaeb, Servicios de Atención Primaria en Salud, Municipalidad de la Pintana, Chile.

${ }^{3}$ Facultad de Odontología, Universidad de los Andes, Santiago, Chile.
\end{abstract}

Autor de correspondencia:

Patricio Felipe Tello Aros

Correos electrónicos: patriciotello90@gmail.com ptelloa@udd.cl
Recibido para publicación:

13 de abril de 2017.

Aceptado para publicación:

13 de junio de 2017. 


\section{INTRODUCCIÓN}

La ansiedad dental es un fenómeno que tiene una importancia significativa en la salud bucal del paciente, teniendo grandes repercusiones en la atención dental y la adherencia a los tratamientos; en países de Europa, la ansiedad dental tiene tasas que varían desde el $4 \%$ al 23\%. En Sudamérica, estos valores alcanzan el $34,7 \%$ en preescolares, adicionalmente se encontró que los padres de estos niños también presentan ansiedad dental en un 41,1\%. En Estados Unidos, cerca de 35 millones de personas padecen ansiedad dental, mientras que solo de 10 a 12 millones de personas sufren de fobia dental, siendo esta el estado más severo de ansiedad ${ }^{1}$.

En la literatura se describen diferentes formas con las cuales se puede guiar el comportamiento de un niño, entre ellas se destacan las guías básicas y avanzadas de comportamiento; es el odontólogo quién determina qué técnica debe ser utilizada, tomando en consideración las necesidades individuales de cada paciente ${ }^{2-6}$, no obstante, dadas las dificultades en el manejo de algunos pacientes, transforman a la sedación en una alternativa indispensable ${ }^{7-12}$.

La sedación consiste es la administración de un agente farmacológico o una combinación de estos, causando alteraciones en el nivel de conciencia, cognición, coordinación motora, nivel de ansiedad y parámetros psicológicos ${ }^{13-16}$. Estas alteraciones son dependientes del fármaco, vía de administración, dosis, y la sensibilidad individual a este o estos agente(s) ${ }^{17-21}$.

Existen tres niveles de sedación: sedación mínima, moderada y profunda. La sedación mínima y moderada se conocen como sedación consciente, en cambio, la sedación profunda como sedación inconsciente ${ }^{11,22-25}$.

Los fármacos comúnmente utilizados en la sedación corresponden a la familia de las benzodiazepinas, no benzodiazepinas, opioides, barbitúricos y bloqueadores de histamina, estos medicamentos se clasifican como sedantes - hipnóticos o ansiolíticos ${ }^{26-27}$.

El efecto que tengan dependerá de la dosis y de la respuesta del paciente, logrando así sedación o un efecto tranquilizante en cantidades bajas, y en dosis altas, hipnosis, que corresponde a un estado similar al que se presenta con el sueño ${ }^{11,26-28}$.

Las benzodiazepinas siguen considerándose como los principales ansiolíticos en farmacoterapia porque son altamente eficaces, comienzan sus efectos terapéuticos rápidamente y tienen un perfil más favorable de efectos colaterales ${ }^{29-31}$. Las benzodiazepinas más utilizadas para la sedación son: Midazolam, Lorazepam y Diazepam ${ }^{22}$.

El Midazolam es frecuentemente utilizado para la premedicación anestésica y sedación debido a que es altamente seguro y efectivo, además, posee una efectiva y rápida difusión a través de la barrera hemato-encefálica, con un inicio de acción a los 20 minutos de su administración. El paciente se puede recuperar rápidamente, ya que cuenta con una vida media corta (de 1 a 4 horas). Usualmente se utiliza en dosis de $0,5 \mathrm{mg} / \mathrm{kg}$ y no requiere uso de soporte ventilatorio ni de oxígeno ${ }^{22-32}$.

\section{MÉTODOS}

Para este trabajo se realizó una revisión bibliográfica de la literatura científica, consultando las siguientes bases de datos electrónicas: PubMed, Scielo, Cochrane Libray, EBSCOhost y Google académico.

Al realizar esta búsqueda se utilizaron palabras claves como: "ansiedad dental", "sedación consciente", "Midazolam", "conscious sedation" y "pediatric dentistry". Con el fín de acceder a los artículos en texto completo se utilizó la base de datos de la Biblioteca Universidad del Desarrollo - UDD y Universidad Católica. Solo se consideraron artículos de revisión, ensayos clínicos y series de casos en texto completo en idiomas inglés y español que fuesen publicados entre 2005 y 2015; no se tuvieron en cuenta reportes de caso para esta revisión bibliográfica.

\section{RESULTADOS}

Después de realizada la búsqueda en las bases electrónicas, de acuerdo con las palabras claves, las restricciones de fecha y los criterios de inclusión y exclusión propuestos, se obtuvieron 78 artículos relacionados con el tema, posteriormente se procedió a leer cada 
resumen de dichos artículos y se seleccionaron 16 estudios para realizar esta revisión bibliográfica.

Los tipos de estudios incluidos en el presente estudio correspondieron a 12 ensayos clínicos, 2 revisiones bibliográficas, 1 revisión sistemática y 1 estudio de series de casos. A continuación se presentarán los resultados obtenidos, los cuales fueron agrupados de acuerdo con la relevancia de los temas tratados en cada uno de ellos. Además para esta agrupación fue considerada la manera en que se midieron las variables en cada estudio de modo que pudieran ser comparables.

\section{Efectividad de la sedación con Midazolam}

En el estudio de Tavassoli-Hojati et al. ${ }^{33}$ del 2014 se hizo una comparación del Midazolam usando dos vías de administración diferentes; oral y bucal o transmucosa. El propósito de este estudio fue investigar la efectividad (medida a través de la escala de Houpt), seguridad y aceptabilidad del Midazolam bucal en comparación con el Midazolam oral, utilizándolo en pacientes odontopediátricos. Se dieron dosis diferentes en relación con la vía de administración, siendo esta $0,3 \mathrm{mg} / \mathrm{kg}$ para el Midazolam bucal y $0,5 \mathrm{mg} / \mathrm{kg}$ para el Midazolam oral.

Se concluyó que el Midazolam bucal y oral tiene resultados similares en cuanto a su efectividad y aceptabilidad. Además, ambos regímenes y vías de administración son considerados seguros sin presentarse rangos anormales de saturación de oxígeno o frecuencia cardíaca (Tabla 1).

En una investigación realizada por Salem et al. ${ }^{34}$ en 2015 se compararon dos formas de presentación de Midazolam oral utilizadas en pacientes niños, una de las variables consideradas en este estudio fue la efectividad de cada preparación.
Estas formas de presentación consistían en un jarabe comercial y una preparación de Midazolam endovenoso administrado oralmente (Midazolam improvisado). Para evaluar la efectividad de cada forma de presentación fue utilizada la escala de Houpt ${ }^{35}$, no encontrándose diferencias estadísticamente significativas entre estas; sin embargo, la administración del jarabe comercial mostró una efectividad incrementada cuando se comparó con el Midazolam improvisado. En relación con esto, la sedación fue efectiva en el 90,9\% de los pacientes en quienes se utilizó el jarabe comercial de Midazolam y en un 79,5\% efectiva de los pacientes sedados con Midazolam improvisado.

En la investigación de Somri et al. ${ }^{36}$ en 2012 se hizo la comparación de 3 dosis diferentes de Midazolam oral, $0,5 \mathrm{mg} / \mathrm{kg}$ (grupo A), $0,75 \mathrm{mg} / \mathrm{kg}$ (grupo B) y $1 \mathrm{mg} / \mathrm{kg}$ (grupo C). El objetivo de este estudio fue optimizar la dosis de este fármaco para la sedación en procedimientos dentales en niños. Se consideraron siete variables, una de ellas fue la efectividad de sedación según la escala de Houpt ${ }^{35}$, dando como resultado diferencias estadísticamente significativas entre el grupo que fue sedado con $0,5 \mathrm{mg} / \mathrm{kg}$ y $1 \mathrm{mg} / \mathrm{kg}$. La sedación se consideró efectiva en el $80 \%$ del grupo A, $96,3 \%$ grupo B y $100 \%$ grupo C.

\section{Dosis, período de latencia y duración del efecto farmacológico del Midazolam}

Numerosos autores ${ }^{33,36-40}$ tomaron en consideración factores como la latencia y duración de la sedación en relación con la dosis de Midazolam administrada.

Se observaron cambios en la latencia dependiendo de la vía de administración, como se puede ver en una investigación hecha por $\mathrm{Mateo}^{39}$, donde se comparó la administración oral e intramuscular en dosis iguales. Al grupo que se le administró Midazolam vía oral el período de latencia fue de 20 minutos, mientras que

Tabla 1. Efectividad, aceptación y seguridad del Midazolam oral y bucal

\begin{tabular}{lcc}
\hline & Midazolam oral 0,5mg/kg & Midazolam bucal 0,3mg/kg \\
\hline Efectividad sedación (éxito) & $88,9 \%$ & $83,4 \%$ \\
Aceptación & $88,8 \%$ & $88,8 \%$ \\
Seguridad (reacciones adversas) & No se presenta & No se presenta \\
\hline
\end{tabular}


al grupo de Midazolam intramuscular fue de $15 \mathrm{mi}-$ nutos. La duración del efecto farmacológico presentó una variación que va desde los 45 minutos a los 117 minutos en los estudios utilizados ${ }^{36,38,40}$ (Tabla 2).

\section{Efectos adversos presentes en sedación}

Aun cuando la sedación se considere como un procedimiento seguro, no está ajeno a presentar reacciones adversas. En el 2012 Lourenço-Matharu, Ashley y Furness ${ }^{41}$ realizaron una revisión sistemática donde se identificó el Midazolam como uno de los pocos fármacos disponibles, cuya eficacia y seguridad para ser utilizado en procedimientos dentales en niños está apoyado por evidencia. Sin embargo, en una revisión realizada por Papineni, Lourenco y Ashley $^{42}$ en 2014 se investigaron los efectos adversos más comunes presentados por este fármaco cuando era utilizado por vía oral. Como conclusión se determinó que la mayor cantidad de efectos adversos reportados eran menores, siendo estos náuseas o vómitos, desaturaciones transitorias y reacciones paradójicas al medicamento.
Somri, et al. ${ }^{36}$, al comparar tres dosis de Midazolam administradas vía oral a 90 pacientes entre 3 y 10 años, observaron diferencias en cuanto a las reacciones adversas presentadas en cada grupo. Los grupos $\mathrm{A}, \mathrm{B}$ y $\mathrm{C}$ recibieron $0,5,0,75 \mathrm{y} 1 \mathrm{mg} / \mathrm{kg}$, respectivamente, cada grupo fue formado por 30 niños. Se presentaron diferencias significativas en la ocurrencia de eventos respiratorios (desaturación de oxígeno $<94 \%$ por más de 30 segundos y maniobras externas para mantener vía aérea permeable), entre el grupo A y C. De igual forma se encontraron diferencias relevantes en los reportes de nauseas entre los grupos A y C, pero no entre A y B o B y C (Tabla 3 ).

En un estudio hecho por Ritwik, Cao, Curran y Musselman ${ }^{43}$ en 2013, se menciona la falta de información en relación con los efectos adversos tardíos presentes en niños que han sido sedados con Midazolam u otros fármacos. El propósito de este estudio fue evaluar y comparar la incidencia de efectos adversos ocurridos a las $8 \mathrm{y}$ a las 24 horas después de la sedación hecha a pacientes odontopediátricos con Meperidina + Hidroxicina v/s Midazolam. Se aplicó una encuesta telefónica a los padres después de 8 y de 24 horas post-sedación (Tabla 4).

Tabla 2. Latencia y duración de la sedación en relación con la dosis utilizada y la vía de administración

\begin{tabular}{lcccc}
\hline Autores (año) & Dosis $\mathbf{~ m g / k g}$ & Latencia (minutos) & Duración (minutos) & Vía administración \\
\hline Ascanio y Núñez $(2009)^{37}$. & 0,75 & 30 & - & Oral \\
\hline Somri et al. $(2012)^{36}$ & 0,75 & $22+-4,3$ & $103,7+-13,3$ & Oral \\
\hline Mateo $(2015)^{39}$ & 0,5 & 20 & - & Oral \\
Mateo $(2015)^{39}$ & 0,5 & 15 & - & Intramuscular \\
Oriolo et al. $(2014)^{40}$ & 0,5 & 15 & 45 & Oral \\
Tavassoli-Hojati et al. $(2014)^{33}$ & 0,5 & $30-45$ & - & Oral \\
Tavassoli-Hojati et al. $(2014)^{33}$ & 0,5 & $10-15$ & 80 & Bucal \\
Bravo et al. $(2009)^{38}$ & 0,4 & 28 & Oral \\
\hline
\end{tabular}

Tabla 3. Reacciones adversas en 3 dosis diferentes de Midazolam vía oral (elaborada con base en los estudios de Somri et al..$^{36}$; Oriolo et al. ${ }^{40}$; Bravo et al. ${ }^{38}$ )

\begin{tabular}{lccc}
\hline & $\mathbf{A}(\mathbf{0}, \mathbf{5 m g} / \mathbf{k g})$ & $\mathbf{B}(\mathbf{0}, \mathbf{7 5} \mathbf{~ \mathbf { g }} / \mathbf{k g})$ & $\mathbf{C}(\mathbf{1} \mathbf{~ m g} / \mathbf{k g})$ \\
\hline Eventos respiratorios & $0 \%$ & $13,3 \%$ & $33,3 \%$ \\
Eventos cardiovasculares & $0 \%$ & $0 \%$ & $0 \%$ \\
Náuseas, mareos & $10 \%$ & $23,3 \%$ & $40 \%$ \\
\hline
\end{tabular}


Tabla 4. Efectos adversos a las 8 y 24 horas luego de sedación con Meperidina + Hidroxicina v/s Midazolam (modificada de Ritwick et al.43)

\begin{tabular}{|c|c|c|}
\hline & $\begin{array}{c}\text { Meperidina+ } \mathrm{Hi}- \\
\text { droxicina } 1-2 \mathrm{mg} / \mathrm{kg}\end{array}$ & $\begin{array}{c}\text { Midazolam } 0,5- \\
0,7 \mathrm{mg} / \mathrm{kg}\end{array}$ \\
\hline \multicolumn{3}{|l|}{ Encuesta 8 horas } \\
\hline Dolor & $35 \%$ & $16,7 \%$ \\
\hline Fiebre & $5 \%$ & $16,7 \%$ \\
\hline Vómitos & $7,5 \%$ & $0 \%$ \\
\hline Duerme en auto & $50 \%$ & $50 \%$ \\
\hline Ronca en auto & $22,5 \%$ & $16,7 \%$ \\
\hline Sueño prolongado & -- & -- \\
\hline Dificultad al despertar & $12,5 \%$ & $0 \%$ \\
\hline Irritable & $5 \%$ & $32 \%$ \\
\hline \multicolumn{3}{|l|}{ Encuesta 24 horas } \\
\hline Dolor & $17,5 \%$ & $16,7 \%$ \\
\hline Fiebre & $0 \%$ & $0 \%$ \\
\hline Vómitos & $2,5 \%$ & $0 \%$ \\
\hline Duerme en auto & -- & -- \\
\hline Ronca en auto & -- & -- \\
\hline Sueño prolongado & $100 \%$ & $66,7 \%$ \\
\hline Dificultad al despertar & $12,5 \%$ & $16,7 \%$ \\
\hline Irritable & $15 \%$ & $16,7 \%$ \\
\hline
\end{tabular}

De esto se concluyó que la mayoría de los efectos adversos tardíos ocurren en las primeras 8 horas, sin embargo, también pueden ocurrir a las 24 horas después de realizada la sedación. La mitad del total de niños sedados durmió en el auto cuando iba de vuelta a casa, esto fue considerado como un riesgo en la obstrucción de la vía aérea. El sueño prolongado fue significativamente menor en los niños sedados con Midazolam.

En una serie de casos hecha por Lee, Milgrom, Starks y Burke ${ }^{44}$ en 2013, se recopiló información sobre muertes causadas por efectos adversos relacionados con sedación y anestesia general en pacientes odontológicos, menores de 21 años, entre 1980 y 2011 en Estados Unidos (Tabla 5).

De lo anterior se concluyó que la mayor cantidad de muertes ocurrieron en niños entre 2 y 5 años; durante exodoncias; en pacientes bajo sedación moderada; dada por un dentista general u odontopediatra en consultas dentales.
Tabla 5. Muertes asociadas con sedación y anestesia general en odontología (traducción de Lee et al.44)

\begin{tabular}{lr}
\hline \multicolumn{1}{c}{ Variables } & n,total=44(\%) \\
\hline Rango de edad: & $2(4,6 \%)$ \\
\hline $0-23$ meses & $21(47,7 \%)$ \\
\hline $2-5$ años & $8(18,2 \%)$ \\
\hline $6-12$ años & $13(29,6 \%)$ \\
\hline $13-21$ años & \\
\hline Procedimiento & $1(2,3 \%)$ \\
\hline Prevención & $14(31,8 \%)$ \\
\hline Restauraciones & $18(40,9 \%)$ \\
\hline Exodoncias & $3(6,8 \%)$ \\
\hline Otras (endodoncias, cirugías) & $8(18,2 \%)$ \\
\hline No reportado & \\
\hline Anestesia/sedación & $4(9,1 \%)$ \\
\hline Anestesia local & $20(45,5 \%)$ \\
\hline Sedación moderada (oral/endovenosa) & $10(22,7 \%)$ \\
\hline Anestesia general & $10(22,7 \%)$ \\
\hline No reportado & \\
\hline Proveedor de anestesia/sedación & $25(56,8 \%)$ \\
\hline Dentista general/odontopediatra & $8(18,2 \%)$ \\
\hline Cirujano oral & $7(15,9 \%)$ \\
\hline Anestesiólogo & $4(9,1 \%)$ \\
\hline No reportado & \\
\hline Lugar & $31(70,5 \%)$ \\
\hline Consulta dental & $6(13,6 \%)$ \\
\hline Centro hospitalario & $7(15,9 \%)$ \\
\hline No reportado & \\
\hline & \\
\hline
\end{tabular}

\section{DISCUSIÓN}

En diferentes estudios ${ }^{33,34,36}$ se alcanzaron tasas de éxito cercanas y mayores al 90\%, lo que comprueba su efectividad a la hora de disminuir la ansiedad en los pacientes. Las diversas tasas de éxito descritas pueden deberse a la variabilidad que presentaron los estudios en cuanto a dosis y vías de administración.

En la literatura se describe que la dosis pediátrica del Midazolam va de 0,2 a $1 \mathrm{mg} / \mathrm{kg}$, con un período de latencia entre 20 y 30 minutos $^{45}$ y una duración promedio entre 60 y 120 minutos.

Con los datos observados no se puede apreciar una directa correlación entre la dosis administrada y el período de latencia del fármaco, sin embargo, estos valores se encuentran dentro del rango normal descrito. 
Para la vía oral el tiempo de latencia fue cercano a los 30 minutos en la mayoría de los estudios. Cuando la dosis aumentaba de igual forma lo hacía su efectividad, sin embargo, también se mostraba un incremento en las reacciones adversas.

Somri, et al. ${ }^{36}$, con el objeto de llegar a un equilibrio entre la efectividad del fármaco y las reacciones adversas presentes, propusieron que dosis entre $0,5 \mathrm{y}$ $0,75 \mathrm{mg} / \mathrm{kg}$ de Midazolam oral eran las óptimas, donde se producía una buena ansiolisis y se permitía llevar el tratamiento dental pediátrico efectivamente, mientras se reducen los efectos adversos respiratorios. Sin embargo, un factor por considerar al momento de determinar la dosis es la edad del paciente, ya que Salem, et al. ${ }^{34}$ menciona que en niños se podrían necesitar dosis más altas, debido a que su hígado tiene más circulación y por lo tanto el metabolismo se ve aumentado, además, poseen menor cantidad de expresión de receptores y sitios de unión por los que tienen afinidad las benzodiazepinas.

En lo que a vías de administración se refiere, también existen variaciones en los resultados obtenidos de acuerdo con la efectividad, esto se explica debido a la poca rigurosidad que existe por parte de los investigadores al momento de administrar Midazolam vía oral. En muchos países no se comercializa el Midazolam oral por lo que en su reemplazo utilizan preparaciones realizadas con base en Midazolam endovenoso mezclado con jugos de fruta o soluciones azucaradas. La diversidad de vehículos utilizados presenta diferencias en cuanto a $\mathrm{pH}$, viscosidad y farmacocinética, lo que podría explicar los diferentes resultados obtenidos en los estudios. Salem, et al. ${ }^{34}$ midieron el $\mathrm{pH}$ de la solución improvisada y del jarabe comercial, estos fueron 3,7 y 4 , respectivamente. Aun cuando estos resultados son similares, se obtuvieron diferencias en cuanto a la efectividad del fármaco. La absorción de fármacos por parte de la mucosa depende del $\mathrm{pH}$, al aumentar el $\mathrm{pH}$ se aumenta la absorción del fármaco en la mucosa. Por lo mencionado anteriormente, el hacer sedación oral con formulaciones comerciales resulta más efectivo, ya que los porcentajes de biodisponibilidad se encuentran determinados.

Cada vía de administración presenta ventajas y desventajas entre las cuales encontramos rapidez de acción en el caso de la vía intranasal, endovenosa, intramuscular o la mayor aceptación que tiene el Midazolam oral en niños siendo considerado como una vía más amigable como fue descrito por Mateo ${ }^{39}$. Para realizar sedación consciente, la vía bucal (transmucosa) parece tener grandes ventajas, como su rápida acción, fácil administración y alta biodisponibilidad, lo que la hace una alternativa interesante, como cuando es utilizado de manera transmucosa en el tratamiento agudo de crisis epilépticas, sin embargo, su aplicación en odontología no es común. A raíz de esto Tavassoli-Hojati et al. ${ }^{33}$, al comparar la utilización de Midazolam oral v/s Midazolam transmucoso en odontología pediátrica, se comprobó un tiempo de latencia menor que el observado durante la administración de forma oral, mayor tasa de éxito en la sedación y seguridad en cuanto a reacciones adversas se refiere, cuando este era aplicado en dosis de $0,3 \mathrm{mg} / \mathrm{Kg}$.

El Midazolam está relativamente libre de presentar algún efecto adverso cuando es administrado en las dosis recomendadas. El mayor riesgo presente con este fármaco es la disminución de la frecuencia respiratoria, lo que provoca una disminución en la saturación de oxígeno; sin embargo, estos efectos se dan cuando es administrado en dosis mayores como se comprobó en un estudio hecho por Somri et al. ${ }^{36}$, en el que esta reacción adversa se presentó en un 33\% de los pacientes a quienes les fue administrado oralmente $1 \mathrm{mg} / \mathrm{kg}$ en contraposición con los pacientes a quienes se les administró en una dosis de $0,5 \mathrm{mg} / \mathrm{kg}$ y no presentaron reacciones adversas de tipo respiratorio. De igual forma sucedió con las náuseas y los vómitos que se produjeron en porcentajes similares.

La depresión respiratoria parece ser un efecto dependiente de la dosis por lo que los regímenes para administrar este fármaco deberían ser seguidos estrictamente. Es fundamental el uso de monitoreo de presión arterial, frecuencia cardíaca, frecuencia respiratoria y saturación de oxígeno a los pacientes que se les administra Midazolam, ya que así es posible advertir de signos tempranos de apnea o hipoventilación. La depresión respiratoria y la disminución de la saturación de oxígeno pueden ser resueltas la mayoría de las veces con estimulación verbal, corrección de la postura de la cabeza para despejar la vía aérea o la administración de presión positiva de oxígeno ${ }^{45}$. 
Por otra parte, la mayoría de los estudios describen las reacciones adversas presentes durante el tratamiento dental o los primeros momentos luego de realizada la sedación, sin embargo, existen pocos estudios donde se mencionen las reacciones adversas tardías más comunes que se tiene en este tipo de pacientes, como fue mencionada por Ritwik et al. ${ }^{43}$, donde las reacciones adversas más graves ocurrieron durante las 8 horas después de sedados con Meperidina más Hidroxicina v/s los sedados con Midazolam. Aun cuando en el grupo sedado con Midazolam las reacciones adversas tardías ocurrieron en menor cantidad, igual estuvieron presentes.

Las conclusiones derivadas de este estudio en cuanto a con qué medicamento la frecuencia de reacciones adversas fue mayor, pueden haber sido poco confiables, debido a la distribución desigual de participantes en cada grupo de estudio. Sin embargo, se decidió considerar de igual forma, por la poca cantidad de evidencia científica disponible donde se describan las reacciones adversas tardías y precauciones principales que se deben tener en estos pacientes.

En el caso de sospechar de una sobredosis por Midazolam existe el Flumazenil, el cual es un antagonista del Midazolam. La seguridad de la sedación con Midazolam ha sido mejorada significativamente por la disponibilidad del Flumazenil, este produce un aumento del estado de alerta y período de recuperación más corto.

\section{CONCLUSIONES}

El Midazolam es una de las benzodiazepinas que más se utiliza por la seguridad de uso, acción rápida y posibilidad de producir amnesia anterógrada en mayor grado que otras benzodiazepinas.

El entrenamiento y equipo apropiado es una necesidad fundamental a la hora de proveer sedación a pacientes de cualquier edad.

La evaluación de cada paciente debe ser exhaustiva, ya que estas prácticas no pueden ser aplicadas de manera estándar.

\section{REFERENCIAS}

1. Alaki S, Alotaibi A, Almabadi E, Alanquri E. Dental anxiety in middle school children and their caregivers: Prevalence and severity. J. Dent. Oral Hyg. 2012;4(1):611. doi: $10.5897 / J D O H 11.019$

2. Gupta A, Marya CM, Bhatia HP, Dahiya V. Behaviour management of an anxious child. Stomatologija. 2014;16(1):3-6.

3. Shahnavaz S, Hedman E, Grindefjord M, Reuterskiöld L, Dahllöf G. Cognitive Behavioral Therapy for Children with Dental Anxiety: A Randomized Controlled Trial. JDR Clinical \& Translational Research. 2016;1(3):234-43. doi: $10.1177 / 238008441666147$

4. Armfield JM, Heaton L. Management of fear and anxiety in the dental clinic: a review. Aust Dent J. 2013;58(4):390407. doi: 10.1111/adj.12118.

5. Peretz B, Nazarian Y, Bimstein E. Dental anxiety in a students' paediatric dental clinic: children, parents and students. International Journal of Paediatric Dentistry. 2004;14(3):192-8. doi: 10.1111/j.1365-263X.2004.00545.x

6. Singh $H$, Rehman R, Kadtane S, Dalai DR, Jain CD. Techniques for the behaviors management in pediatric dentistry. International Journal of Scientific Study. 2014;2(7):269-72.

7. McWhorter AG. Treatment planning for the pediatric patient. Tex Dent J. 2010;127(12):1275-80.

8. Walley S, Albadri S. Undergraduates' perceptions of the value of practical inhalation sedation experience in a UK dental school. Eur Arch Paediatr Dent. 2015;16(5):371-6. doi: 10.1007/s40368-014-0173-5.

9. Wilson S. Management of child patient behavior: quality of care, fear and anxiety, and the child patient. J Endod. 2013;39(3):S73-7. doi: 10.1016/j.joen.2012.11.040.

10. Rojano-Santillán A, Martínez-Ruíz V, Pizano-Damasco M, Banderas-Tarabay J. Dosis efectiva de Midazolam para sedación consciente en estomatología pediátrica. Revista ADM. 2004;61(4):130-136.

11. Shavlokhova EA, Ostreikov IF, Korolenkova MV. Sedation with midazolam for ambulatory pediatric dentistry. Anesteziol Reanimatol. 2014;59(5):48-52.

12. Oliver K, Manton DJ. Contemporary behavior management techniques in clinical pediatric dentistry: out with the old and in with the new?. J Dent Child. 2015;82(1):22-8. 
13. Kupietzky A, Houpt MI. Midazolam: A review of its use for conscious sedation in children. Pediatr Dent. 1993;15:237.

14. Abed HH. Practical Conscious Sedation. Saudi Med J. 2017;38(7):772.

15. Siddiqui F. Anesthesia-related mortality in pediatric patients in dental office setting. J Indian Soc Pedod Prev Dent. 2017;35(2):184. doi: 10.4103/JISPPD. JISPPD_108_16.

16. Jackson DL, Johnson BS. Conscious sedation for dentistry: risk management and patient selection. Dent Clin North Am. 2002;46(4):767-80. doi: 10.1016/ S0011-8532(02)00034-4

17. Hosey M, UK National Clinical Guidelines in Pediatric Dentistry. Managing anxious children: the use of conscious sedation in paediatric dentistry. International Journal of Paediatric Dentistry. 2002;12(5):359-72. doi: 10.1046/j.1365-263X.2002.03791.x

18. Yonel Z, Asuni A, Taneja P. Defining Over-Sedation: Literature Review and National Survey of Dental Hospitals Within the United Kingdom. SAAD Dig. 2016;32:28-33.

19. American Academy of Pediatrics, Cote CJ, Wilson S, Work Group on Sedation. Guidelines for monitoring and management of pediatric patients during and after sedation for diagnostic and therapeutic procedures: an update. Pediatrics. 2006;118(6):2587-602.

20. Yagiela JA, Cote CJ, Notterman DA, Karl HW, Weinberg JA, McCloskey C. Adverse sedation events in pediatrics. Pediatrics. 2001;107(6):1494. doi: 10.1542/ peds.107.6.1494

21. Attri JP, Sharan R, Makkar V, Gupta KK, Khetarpal R, Kataria AP. Conscious sedation: Emerging trends in pediatric dentistry. Anesthesia: Essays and Researches. 2017;11(2):277. doi: 10.4103/0259-1162.171458.

22. Álvarez AM, Álvarez M. Sedación oral: fundamentos clínicos para su aplicación en odontología. Revista CES Odontología. 2006;19(2):61-73.

23. Beach ML, Cohen DM, Gallagher SM, Cravero JP. Major Adverse Events and Relationship to "Nil per Os" Status in Pediatric Sedation/Anesthesia Outside the Operating Room: A Report of the Pediatric Sedation Research Consortium. Surv Anesthesiol. 2016;60(5):203. doi: 10.1097/ ALN.0000000000000933.

24. Coulthard P. Conscious sedation guidance. Evid Based Dent. 2006;7(4):90. doi:10.1038/sj.ebd.6400441.
25. Sheahan C, Mathews D. Monitoring and delivery of sedation. Br J Anaesth. 2014;113(2):ii37-ii47. doi: 10.1093/ bja/aeu378.

26. Seymour RA. Pharmacology and therapeutics in dentistry. Periodontol. 2000 2008;46(1):7-8. doi:10.1111/j.1600-0757.2008.00237.x

27. Somri M, Matter I, Hadjittofi C, Hoash N, Moaddi B, Kharouba J et al. Detection of Respiratory Adverse Events in Pediatric Dental Patients Sedated With 0.75 $\mathrm{mg} / \mathrm{Kg}$ of Midazolam and Oxygen by Continuous Pretracheal Auscultation: A Prospective Randomized Controlled Trial. J Clin Pediatr Dent. 2017;41(2):154-60. doi: 10.17796/1053-4628-41.2.154.

28. Gentz R, Casamassimo P, Amini H, Claman D, Smiley M. Safety and Efficacy of 3 Pediatric Midazolam Moderate Sedation Regimens. Anesth Prog. 2017;64(2):66-72. doi: 10.2344/anpr-64-02-04.

29. López-Vantour A, Aroche-Arzuaga A, Bestard-Romero J, Ocaña-Fontela N. Uso y abuso de las benzodiazepinas. Medisan. 2010;14(4):555-66.

30. Kuang H, Johnson JA, Mulqueen JM, Bloch MH. The efficacy of benzodiazepines as acute anxiolytics in children: A meta-analysis. Depression and Anxiety. 2017;34(10):88896 doi: $10.1002 /$ da.22643.

31. Buffett-Jerrott S, Stewart S. Cognitive and sedative effects of benzodiazepine use. Curr Pharm Des. 2002;8(1):45-58. doi: $10.2174 / 1381612023396654$.

32. McMillan C, Spahr-Schopfer I, Sikich N, Hartley E, Lerman J. Premedication of children with oral midazolam. Canadian Journal of Anaesthesia. 1992;39(6):545-50.

33. Tavassoli-Hojjati S, Mehran M, Haghgoo R, Tohid-Rahbari M, Ahmadi R. Comparison of oral and buccal midazolam for pediatric dental sedation: a randomized, crossover, clinical trial for efficacy, acceptance and safety. Iran J Pediatr. 2014;24(2):198-206.

34. Salem K, Kamranzadeh S, Kousha M, Shaeghi S, AbdollahGorgi F. Two Oral Midazolam Preparations in Pediatric Dental Patients: A Prospective Randomised Clinical Trial. Int J Pediatr. 2015;2015:1-7. doi: $10.1155 / 2015 / 349795$.

35. Houpt MI, Rosivack RG, Rozenfarb N, Koenigsberg SR. Effects of nitrous oxide on chloral hydrate sedation of young children. Anesth Prog. 1986;33(6):298-302.

36. Somri M, Parisinos CA, Kharouba J, Cherni N, Smidt A, Abu Ras Z et al. Optimising the dose of oral midazolam sedation for dental procedures in children: a pros- 
pective, randomised, and controlled study. International Journal of Paediatric Dentistry. 2012;22(4):271-279. doi: 10.1111/j.1365-263X.2011.01192.x.

37. Ascanio K, Núñez M. Sedación consciente en tratamiento odontológico: Efectividad de dos medicaciones en niños. Revista Científica Odontología Sanmarquina. Odontología Sanmarquina. 2009;12(2):52-6.

38. Bravo M, Flores Z, Barradas V. Operatoria dental con sedación en preescolares. Revista de la Asociación Dental Mexicana. 2009;66(3):34-9.

39. Mateo M. Premedicación anestésica con Midazolam en niños en el Hospital Regional de Ica, Perú. 2007-2008. Revista Médica Panacea. 2015;2(2):40-4.

40. Oriolo R, Sanabria JG, Oriolo L. Eficacia del Midazolam contra la ansiedad en niños de 1-3 años sometidos a cirugía. Revista de Ciencias Médicas de Pinar del Río. 2014;18(3):388-400.
41. 41. Lourenço-Matharu L, Ashley PF, Furness S. Sedation of children undergoing dental treatment. The Cochrane Library. 2012;(3):1-105. doi: 10.1002/14651858. CD003877.

42. Papineni A, Lourenço-Matharu L, Ashley PF. Safety of oral midazolam sedation use in paediatric dentistry: a review. International Journal of Paediatric Dentistry. 2014;24(1):2-13. doi: 10.1111/ipd.12017.

43. Ritwik P, Cao LT, Curran R, Musselman RJ. Post-sedation events in children sedated for dental care. Anesth Prog. 2013;60(2):54-9. doi: 10.2344/0003-3006-60.2.54.

44. Lee HH, Milgrom P, Starks H, Burke W. Trends in death associated with pediatric dental sedation and general anesthesia. Pediatric Anesthesia. 2013;23(8):741-6. doi: 10.1111/pan.12210.

45. Alzahrani AM, Wyne AH. Use of oral Midazolam sedation in pediatric dentistry: a Review. Pakistan Oral \& Dental Journal. 2012;32(3):444-5. 